\title{
Monocyte to lymphocyte ratio predicts survival in patients with advanced gastric cancer undergoing neoadjuvant chemotherapy
}

\author{
Li Chen ${ }^{1, *}$ \\ Ying $\mathrm{Hao}^{2, *}$ \\ Lihua Zhu ${ }^{3}$ \\ Sen $\mathrm{Li}^{\prime}$ \\ Yanjiao Zuo' \\ Yuxin Zhang ${ }^{4}$ \\ Hongjiang Song' \\ Yingwei Xue' \\ 'Department of Gastrointestinal \\ Surgery, Harbin Medical University \\ Cancer Hospital, Harbin Medical \\ University, Harbin, Heilongjiang, China; \\ ${ }^{2}$ Department of Internal Oncology, \\ Harbin The First Hospital, Harbin, \\ Heilongjiang, China; ${ }^{3}$ Department of \\ Pathogen Biology, School of Basic \\ Medical Sciences, North China \\ University of Science and Technology, \\ Tangshan, Hebei, China; ${ }^{4}$ Department \\ of General Surgery, Mudanjiang \\ First People's Hospital, Mudanjiang, \\ Heilongjiang, China \\ *These authors contributed equally \\ to this work
}

This article was published in the following Dove Press journal:

OncoTargets and Therapy

10 August 2017

Number of times this article has been viewed

Background: Currently, precise predictors in gastric cancer patients undergoing neoadjuvant chemotherapy are lacking. The study aims to investigate the prognostic value of the monocyte to lymphocyte ratio (MLR) in patients with advanced gastric cancer receiving S-1 plus oxaliplatin (SOX) or oxaliplatin and capecitabine (XELOX) neoadjuvant chemotherapy regimen.

Methods: The data from Harbin Medical University Cancer Hospital from August 2008 to September 2015 were retrospectively collected. Ninety-one patients with advanced gastric cancer treated with neoadjuvant chemotherapy were included. The blood samples were collected before neoadjuvant chemotherapy. The MLR was divided into two groups: Low-MLR $<0.27$ group and high-MLR $\geq 0.27$ group. Survival curves were performed using the Kaplan-Meier method and compared using the log-rank test. Univariate and multivariate Cox proportional hazards regression model were evaluated to determine independent prognostic factors.

Results: The univariate analysis showed that median disease free survival (DFS) and overall survival (OS) for all patients were better in low-MLR value group than high-MLR value group (median DFS 26.80 and 23.73 months, $P=0.653$, respectively; median OS 27.93 and 26.87 months, $P=0.807$, respectively). Multivariate analysis showed that MLR level was not an independent prognostic factor of DFS and OS. Nevertheless, median DFS and OS for all patients were better for patients with low monocyte values compared to those with high monocyte values (median DFS 30.23 and 21.03 months, $P=0.645$, respectively; median OS 37.97 and 25.83 months, $P=0.509$, respectively); in patients with high lymphocyte values compared with low lymphocyte values median DFS was 26.87 and 21.03 months, $(P=0.624)$ respectively; median OS was 27.93 and 26.37 months, $(P=0.584)$ respectively. However, the patients with low level MLR had better 5-year DFS and OS rates.

Conclusion: MLR may be used as a convenient and cheap prognostic marker in patients with advanced gastric cancer undergoing neoadjuvant chemotherapy with SOX or XELOX. Low level MLR as a prognostic marker may help doctors in terms of efficient measures to treat gastric cancer.

Keywords: neoadjuvant chemotherapy, advanced gastric cancer, monocyte to lymphocyte ratio (MLR), disease free survival, overall survival

Department of Gastrointestinal Surgery, Harbin Medical University Cancer Hospital, Harbin Medical University, Harbin, Heilongjiang I5008I, China Email hongjiangsong2016@163.com

Lihua Zhu

Department of Pathogen Biology, School of Basic Medical Sciences, North China University of Science and Technology, Tangshan, Hebei 063000, China Email zhulihua1972@I63.com

\section{Introduction}

Gastric cancer is the most common type of digestive system malignant tumors and the main cause of death in the whole world. ${ }^{1}$ Surgical operation is the major treatment for advanced gastric cancer. However, recurrence and metastasis are common factors leading to low level 5-year survival rate in gastric cancer. In China, advanced gastric carcinoma accounts for the majority of all patients with gastric cancer, and 
the preoperative clinical tumor stage is usefulness to choose the better therapeutic strategy for patients. Nevertheless, it may not predict patients' postoperative complications and prognosis. Gastric cancer death could be prevented if the disease was detected earlier. Hence, it is significant to explore actively the potential prognostic markers that could predict the survival of gastric carcinoma patients at high risk of recurrence and metastasis.

For several decades, neoadjuvant chemotherapy has been shown to be beneficial in the treatment of advanced gastric cancer. Many studies have demonstrated that the neoadjuvant chemotherapy may either decrease the tumor stage or increase the $\mathrm{R} 0$ resection rate without increasing surgical morbidity and mortality. ${ }^{2}$ The neoadjuvant chemotherapy may result in increasing pathological complete response (CR) with tolerable side effects, and decreasing positive pathological nodes. ${ }^{3}$ Many neoadjuvant chemotherapy regimens have been used for gastric cancer treatment, and the S-1 plus oxaliplatin (SOX) and oxaliplatin and capecitabine (XELOX) regimens are the major regimens in clinical practice. ${ }^{4}$ In Asia, with these neoadjuvant chemotherapy regimens and surgical resection, it has remarkably improved survival of patients with advanced gastric cancer. ${ }^{5}$ Hence, it is crucial to find new tumor indicators to improve better survival outcome. ${ }^{6}$

Inflammation is the fundamental component of the tumor microenvironment, and the change of inflammatory cells might affect tumor progression, such as tumor cell proliferation, migration, invasion, metastasis, and so forth. ${ }^{7,8}$ In recent years, the relationship between inflammation and malignant tumor has been hotly studied. Tumor-inflammation interaction may be a potential therapeutic target for the neoplastic treatment, and the peripheral blood tests may influence and reflect the tumor inflammatory conditions. Many studies have shown that $\mathrm{C}$-reactive protein (CRP), white blood cell (WBC), neutrophil, lymphocyte, monocyte, and platelet counts, as well as neutrophil to lymphocyte ratio (NLR), derived neutrophil to lymphocyte ratio (d-NLR), platelet to lymphocyte ratio (PLR), and monocyte to lymphocyte ratio (MLR) were used in many malignancies to quantify the survival outcome. ${ }^{9,10}$

As far as we know, the MLR has been identified as a useful predictor in gastric carcinoma. ${ }^{11}$ However, the prognostic value of MLR in patients receiving the SOX or XELOX regimen for advanced gastric carcinoma is rarely reported. Therefore, the aim of our study was to evaluate the prognostic value of MLR in patients with advanced gastric cancer receiving SOX or XELOX neoadjuvant chemotherapy regimen.

\section{Materials and methods Ethics approval}

This study was approved by the ethics committee of the Harbin Medical University Cancer Hospital, Harbin Medical University and all procedures were performed in accordance with the standards of the Declaration of Helsinki. All human materials were obtained with written informed consent.

\section{Patient selection}

We performed a retrospective analysis of a clinicopathological database at Harbin Medical University Cancer Hospital, Harbin Medical University. All patients with stage II/III gastric carcinoma and treated with neoadjuvant chemotherapy between August 2008 to September 2015 were included. All cases were diagnosed with pathological evidence, and the clinical stage was determined as II/III according to tumornode-metastasis (TNM) staging system. ${ }^{12}$ Patients were accepted into this study if they satisfied the following criteria: 1) all patients were diagnosed with locally advanced gastric cancer by pathological evidence; 2) Eastern Cooperative Oncology Group performance status with the range being 0-2, Karnofsky performance status must be $\geq 80$; 3) a life expectancy survival of 3 months or longer; 4) no history of chemotherapy, radiotherapy, targeted therapy, and so forth. Patients were excluded if they met one of the following criteria: 1) patient was found to have distant metastases; 2) patient had some acute or chronic disease, such as diabetes mellitus, hypertension, atherosclerotic heart diseases, and difficult to control; 3) patients with obvious infections, active bleeding, intestinal obstruction; 4) patient received a blood transfusion within 1 month before neoadjuvant chemotherapy.

\section{Treatment protocols}

SOX regimen: oxaliplatin (Jiangsu Hengrui Medicine Co., Ltd., Lianyungang, China) at $130 \mathrm{mg} / \mathrm{m}^{2}$ (intravenous infusion administered in $500 \mathrm{~mL}$ of $5 \%$ glucose over a period of $2 \mathrm{~h}$ ) and S-1 (Taiho Pharmaceutical Co., Ltd., Tokyo, Japan) of $60 \mathrm{mg}$ (orally administered twice a day on days 1-14). XELOX regimen: oxaliplatin at $130 \mathrm{mg} / \mathrm{m}^{2}$ (intravenous infusion administered in $500 \mathrm{~mL}$ of $5 \%$ glucose over a period of $2 \mathrm{~h}$ ) and capecitabine (Shanghai Roche Pharmaceuticals Ltd., Shanghai, China) of 1,500 mg (orally administered twice a day on days 1-14). A cycle of the two regimens were repeated every 3 weeks.

\section{Response evaluation}

Response rates were determined according to the Response Evaluation Criteria In Solid Tumors (RECIST) guidelines ${ }^{13}$ and included the following categories: $\mathrm{CR}$, partial response 
(PR), stable disease (SD), and progression of disease (PD). The clinical objective response rate was calculated as the sum of $\mathrm{CR}$ and $\mathrm{PR}$, and non-clinical response was calculated as the sum of SD and PD.

\section{Blood parameters}

Blood samples were taken at the time of diagnosis before neoadjuvant chemotherapy. WBC differential counts were analyzed by XE-2100 hematology analyzer (Sysmex, Kobe, Japan).

\section{Follow-up}

After surgery, all patients were monitored every 3 months for the first 2 years, every 6 months thereafter, then annually, and until death. The patients were followed regularly every year thereafter with laboratory tests, multi-slice computed tomography (CT), and gastroscopy. Disease free survival (DFS) was measured from the date of surgery until date of relapse (local recurrence and distant metastases). Overall survival (OS) was measured from the date of surgery until date of death from any cause or the last follow-up. The last follow-up date was December 3, 2016.

\section{Statistical analysis}

Statistical analyses were performed using SPSS software (version 17.0; SPSS Inc., Chicago, IL, USA). The optimal cutoff value was determined using receiver operating characteristic (ROC) curve analyses. The area under the curve (AUC) was used to assess the predictive value. The categorical variables of the clinicopathological database of the patients were assessed by the $\chi^{2}$ test or Fisher's exact test. The qualitative variables were expressed as the mean \pm standard error, and compared using Student's $t$-test. Survival curves were performed using the Kaplan-Meier method and compared using the log-rank test. Univariate and multivariate Cox proportional hazards regression model was evaluated to determine independent prognostic factors. All tests were two-sided, and a two-tailed $P<0.05$ was considered to indicate statistical significance.

\section{Results}

\section{Patient characteristics}

We used the ROC curve to determine an optimal cut-off value of the MLR. The AUC of MLR was 0.553, and the optimal cut-off value was 0.27 . The patients were divided into two groups by the optimal cut-off value of MLR (low-MLR $<0.27$, high-MLR $\geq 0.27$ ). We enrolled 91 patients in this retrospective study. Of the patients, $76.9 \%$ (70/91) were males and $23.1 \%(21 / 91)$ of the patients were females; the median age of all patients was 57 years (range: $32-73$ years), with a median body mass index of
22.32 (range: 17.06-34.08). A low baseline MLR level (low-MLR <0.27) correlated with improved demographic and clinicopathological characteristics, including gender $\left(\chi^{2}=4.622, P\right.$-value $\left.=0.032\right)$, primary tumor site $\left(\chi^{2}=8.078\right.$, $P$-value $=0.011)$, monocyte $\left(\chi^{2}=15.072, P\right.$-value $\left.<0.001\right)$, lymphocyte $\left(\chi^{2}=8.315, P\right.$-value $\left.=0.004\right)($ Table 1$)$.

\section{Survival analyses}

The median DFS for all the patients was 23.73 months (range 1.17-93.87 months), and the median OS for all the patients was 26.87 months (range 4.03-96.00 months) (Figures 1 and 2). Regarding DFS, high DFS factors were predicted for $\mathrm{R} 0$ resection, primary tumor site, pathological tumor in situ (Tis)/I stage. In multivariate Cox regression analysis, high DFS factors were predicted for R0 resection ( $P$-value $<0.001$, HR: 2.059, 95\% CI: 1.445-2.932), pathological Tis/I stage $(P$-value $<0.001, \mathrm{HR}: 2.782,95 \% \mathrm{CI}$ : 1.829-4.233) (Table 2). High OS factors were predicted for $\mathrm{R} 0$ resection, pathological Tis/I stage. In multivariate Cox regression analysis, high OS factors were predicted for R0 resection $(P$-value $<0.001$, HR: $2.494,95 \% \mathrm{CI}$ : $1.730-3.595)$, pathological Tis/I stage $(P$-value $<0.001$, HR: 3.041, 95\% CI: 1.949-4.746) (Table 3).

Moreover, we found that monocyte count, lymphocyte count, and MLR before neoadjuvant chemotherapy had no prognostic significance using the cut-off values of $0.44 \times 10^{9} / \mathrm{L}$, $1.68 \times 10^{9} / \mathrm{L}$, and 0.27 regarding DFS $(P$-value $=0.365,0.240$, 0.938 , respectively) and OS $(P$-value $=0.191,0.212,0.904$, respectively) in univariate analysis (Tables 2 and 3). Nevertheless, median DFS and OS for all patients were better for advanced gastric cancer patients with low MLR values than for those with high MLR values (median DFS 26.80 and 23.73 months, $P=0.653$, respectively; median OS 27.93 and 26.87 months, $P=0.807$, respectively) (Figures 3 and 4).

At the same time, we found that median DFS and OS for all patients were better for advanced gastric cancer patients with low monocyte values than for those with high monocyte values (median DFS 30.23 and 21.03 months, $P=0.645$, respectively; median OS 37.97 and 25.83 months, $P=0.509$, respectively) (Figures 5 and 6). Median DFS and OS for all patients were better for advanced gastric cancer patients with high lymphocyte values than for those with low lymphocyte values (median DFS 26.87 and 21.03 months, $P=0.624$, respectively; median OS 27.93 and 26.37 months, $P=0.584$, respectively) (Figures 7 and 8; Table 4).

The patients with low level MLR and low level monocyte count had better median DFS and OS (mean DFS 36.30 and OS 48.21 months, respectively); patients with low level MLR and high level lymphocyte count had better median DFS and 
Table I Demographic and clinicopathological characteristics of 91 patients with advanced gastric cancer

\begin{tabular}{|c|c|c|c|c|c|}
\hline Parameters & Patients & Low-MLR $<0.27$ & High-MLR $\geq 0.27$ & $\chi^{2}$ & $P$-value \\
\hline Age (years) & & & & $\mathrm{I} .846$ & 0.174 \\
\hline$<57$ & 45 (49.5\%) & $24(57.1 \%)$ & $21(42.9 \%)$ & & \\
\hline$\geq 57$ & $46(50.5 \%)$ & $18(42.9 \%)$ & $28(57.1 \%)$ & & \\
\hline Gender & & & & 4.622 & 0.032 \\
\hline Male & 70 (76.9\%) & $28(66.7 \%)$ & $42(85.7 \%)$ & & \\
\hline Female & $21(23.1 \%)$ & $14(33.3 \%)$ & $7(14.3 \%)$ & & \\
\hline BMI $\left(\mathrm{kg} / \mathrm{m}^{2}\right)$ & & & & 1.357 & 0.244 \\
\hline$<22.32$ & 45 (49.5\%) & 18 (42.9\%) & 27 (55.1\%) & & \\
\hline$\geq 22.32$ & $46(50.5 \%)$ & $24(57.1 \%)$ & 22 (44.9\%) & & \\
\hline ABO blood type & & & & 2.797 & $0.473^{\#}$ \\
\hline A & $23(25.3 \%)$ & $12(28.6 \%)$ & II (22.4\%) & & \\
\hline B & $32(35.2 \%)$ & $14(33.3 \%)$ & $18(36.7 \%)$ & & \\
\hline O & $27(29.7 \%)$ & $10(23.8 \%)$ & $17(34.7 \%)$ & & \\
\hline$A B$ & $9(9.9 \%)$ & $6(14.3 \%)$ & $3(6.1 \%)$ & & \\
\hline Chemotherapy regimen & & & & $\mathrm{I} .858$ & 0.173 \\
\hline sox & 35 (38.5\%) & $13(31.0 \%)$ & $22(44.9 \%)$ & & \\
\hline XELOX & $56(61.5 \%)$ & 29 (69.0\%) & $27(55.1 \%)$ & & \\
\hline Radical resection & & & & 2.460 & 0.292 \\
\hline Ro & $5 \mathrm{I}(56.0 \%)$ & $24(57.1 \%)$ & $27(55.1 \%)$ & & \\
\hline RI & $21(23.1 \%)$ & $7(16.7 \%)$ & $14(28.6 \%)$ & & \\
\hline $\mathrm{R} 2$ & 19 (20.9\%) & II (26.2\%) & $8(16.3 \%)$ & & \\
\hline Type of surgery & & & & 2.802 & $0.330^{\#}$ \\
\hline Distal gastrectomy & $52(57.1 \%)$ & 27 (64.3\%) & $25(51.0 \%)$ & & \\
\hline Proximal gastrectomy & $6(6.6 \%)$ & I (2.4\%) & $5(10.2 \%)$ & & \\
\hline Total gastrectomy & $33(36.3 \%)$ & $14(33.3 \%)$ & $19(38.8 \%)$ & & \\
\hline Differentiation & & & & 1.927 & $0.462^{\#}$ \\
\hline Poorly differentiated & $54(59.3 \%)$ & $24(57.1 \%)$ & $30(61.2 \%)$ & & \\
\hline Moderately differentiated & $32(35.2 \%)$ & 17 (40.5\%) & $15(30.6 \%)$ & & \\
\hline Well differentiated & $5(5.5 \%)$ & I (2.4\%) & $4(8.2 \%)$ & & \\
\hline Primary tumor site & & & & 8.078 & $0.011^{\#}$ \\
\hline Upper I/3 & II (12.1\%) & $2(4.8 \%)$ & $9(18.4 \%)$ & & \\
\hline Middle I/3 & 31 (34.1\%) & II (26.2\%) & $20(40.8 \%)$ & & \\
\hline Low $1 / 3$ & $49(53.8 \%)$ & $29(69.0 \%)$ & $20(40.8 \%)$ & & \\
\hline Pathology & & & & 1.600 & $0.714^{\#}$ \\
\hline Adenocarcinoma & $64(70.3 \%)$ & $31(73.8 \%)$ & $33(67.3 \%)$ & & \\
\hline Mucinous carcinoma & $10(11.0 \%)$ & $5(11.9 \%)$ & $5(10.2 \%)$ & & \\
\hline Signet ring cell carcinoma & $12(13.2 \%)$ & $5(11.9 \%)$ & 7 (14.3\%) & & \\
\hline Others & $5(5.5 \%)$ & I (2.4\%) & $4(8.2 \%)$ & & \\
\hline \multicolumn{6}{|l|}{ Clinical TNM classification } \\
\hline T stage & & & & 0.038 & 1.000 \\
\hline $\mathrm{T} 3$ & $6(6.6 \%)$ & $3(7.1 \%)$ & $3(6.1 \%)$ & & \\
\hline $\mathrm{T} 4$ & $85(93.4 \%)$ & $39(92.9 \%)$ & $46(93.9 \%)$ & & \\
\hline $\mathrm{N}$ stage & & & & 0.055 & 0.973 \\
\hline No & $24(26.4 \%)$ & II (26.2\%) & $13(26.5 \%)$ & & \\
\hline NI & $5 \mathrm{I}(56.0 \%)$ & $24(57.1 \%)$ & $27(55.1 \%)$ & & \\
\hline N2 & $16(17.6 \%)$ & $7(16.7 \%)$ & $9(18.4 \%)$ & & \\
\hline TNM stage & & & & - & $1.000^{\#}$ \\
\hline$\|$ & $2(2.2 \%)$ & I (2.4\%) & I (2.0\%) & & \\
\hline III & $89(97.8 \%)$ & $4 \mathrm{I}(97.6 \%)$ & 48 (98.0\%) & & \\
\hline \multicolumn{6}{|l|}{ Pathological TNM classification } \\
\hline T stage & & & & 5.895 & $0.176^{\#}$ \\
\hline TO & $5(5.5 \%)$ & I (2.4\%) & $4(8.2 \%)$ & & \\
\hline $\mathrm{TI}$ & $7(7.7 \%)$ & $4(9.5 \%)$ & $3(6.1 \%)$ & & \\
\hline $\mathrm{T} 2$ & $14(15.4 \%)$ & $10(23.8 \%)$ & $4(8.2 \%)$ & & \\
\hline T3 & $43(47.3 \%)$ & 17 (40.5\%) & $26(53.1 \%)$ & & \\
\hline $\mathrm{T} 4$ & $22(24.2 \%)$ & $10(23.8 \%)$ & $12(24.5 \%)$ & & \\
\hline
\end{tabular}


Table I (Continued)

\begin{tabular}{|c|c|c|c|c|c|}
\hline Parameters & Patients & Low-MLR $<0.27$ & High-MLR $\geq 0.27$ & $\chi^{2}$ & $P$-value \\
\hline $\mathrm{N}$ stage & & & & 0.121 & 0.989 \\
\hline No & $24(26.4 \%)$ & II (26.2\%) & 13 (26.5\%) & & \\
\hline NI & $23(25.3 \%)$ & $10(23.8 \%)$ & $13(26.5 \%)$ & & \\
\hline N2 & 15 (16.5\%) & $7(16.7 \%)$ & $8(16.3 \%)$ & & \\
\hline N3 & $29(31.9 \%)$ & $14(33.3 \%)$ & $15(30.6 \%)$ & & \\
\hline TNM stage & & & & 3.039 & $0.547^{\#}$ \\
\hline Tis & $5(5.5 \%)$ & I (2.4\%) & $4(8.2 \%)$ & & \\
\hline I & 9 (9.9\%) & $6(14.3 \%)$ & $3(6.1 \%)$ & & \\
\hline II & $29(31.9 \%)$ & $13(31.0 \%)$ & $16(32.7 \%)$ & & \\
\hline III & 45 (49.5\%) & 21 (50.0\%) & $24(49.0 \%)$ & & \\
\hline IV & $3(3.3 \%)$ & I $(2.4 \%)$ & $2(4.1 \%)$ & & \\
\hline Total lymph nodes & & & & 0.554 & 0.457 \\
\hline$<27$ & 45 (49.5\%) & 19 (45.2\%) & $26(53.1 \%)$ & & \\
\hline$\geq 27$ & $46(50.5 \%)$ & $23(54.8 \%)$ & $23(46.9 \%)$ & & \\
\hline Positive lymph nodes & & & & 0.017 & 0.897 \\
\hline$<3$ & $44(48.4 \%)$ & 20 (47.6\%) & $24(49.0 \%)$ & & \\
\hline$\geq 3$ & $47(51.6 \%)$ & $22(52.4 \%)$ & $25(51.0 \%)$ & & \\
\hline HER-2 & & & & 0.791 & 0.374 \\
\hline 0 or + & $54(59.3 \%)$ & 27 (64.3\%) & $27(55.1 \%)$ & & \\
\hline++ or +++ & $37(40.7 \%)$ & $15(35.7 \%)$ & $22(44.95)$ & & \\
\hline Monocyte & & & & 15.072 & $<0.001$ \\
\hline$<0.44 \times 10^{9} / \mathrm{L}$ & 45 (49.5\%) & 30 (71.4\%) & 15 (30.6\%) & & \\
\hline$\geq 0.44 \times 10^{9} / \mathrm{L}$ & $46(50.5 \%)$ & $12(28.6 \%)$ & 34 (69.4\%) & & \\
\hline Lymphocyte & & & & 8.315 & 0.004 \\
\hline$<1.68 \times 10^{9} / \mathrm{L}$ & $43(47.3 \%)$ & $13(31.0 \%)$ & 30 (6I.2\%) & & \\
\hline$\geq 1.68 \times 10^{9} / \mathrm{L}$ & $48(52.7 \%)$ & 29 (69.0\%) & $19(38.8 \%)$ & & \\
\hline Response & & & & $3.97 \mid$ & $0.275^{\#}$ \\
\hline CR & $5(5.5 \%)$ & I (2.4\%) & $4(8.2 \%)$ & & \\
\hline PR & 65 (71.4\%) & 34 (81.0\%) & $31(63.3 \%)$ & & \\
\hline SD & 7 (7.7\%) & $3(7.1 \%)$ & $4(8.2 \%)$ & & \\
\hline PD & 14 (I5.4\%) & $4(9.5 \%)$ & $10(20.4 \%)$ & & \\
\hline
\end{tabular}

Note: \#Performed using the Fisher's exact test.

Abbreviations: MLR, monocyte to lymphocyte ratio; BMI, body mass index; SOX, S-I plus oxaliplatin; XELOX, oxaliplatin and capecitabine; TNM, tumor-node-metastasis; $\mathrm{CR}$, complete response; $\mathrm{PR}$, partial response; $\mathrm{SD}$, stable disease; PD, progression of disease; Tis, tumor in situ.

OS (median DFS 36.93 and OS 39.93 months, respectively) (Tables 5 and 6).

The 1-year, 3-year, and 5-year DFS and OS rates for all patients with advanced gastric cancer were $75.8 \%, 23.1 \%$,



Figure I Disease free survival of 91 patients with advanced gastric cancer.
$7.7 \%, 87.9 \%, 26.4 \%$, and $11.0 \%$, respectively. The patients with low MLR $<0.27$ had better 5-year DFS and OS rates; compared with those with high MLR $\geq 0.27$, the 1 -year and 3 -year DFS and OS rates were similar (Table 7).

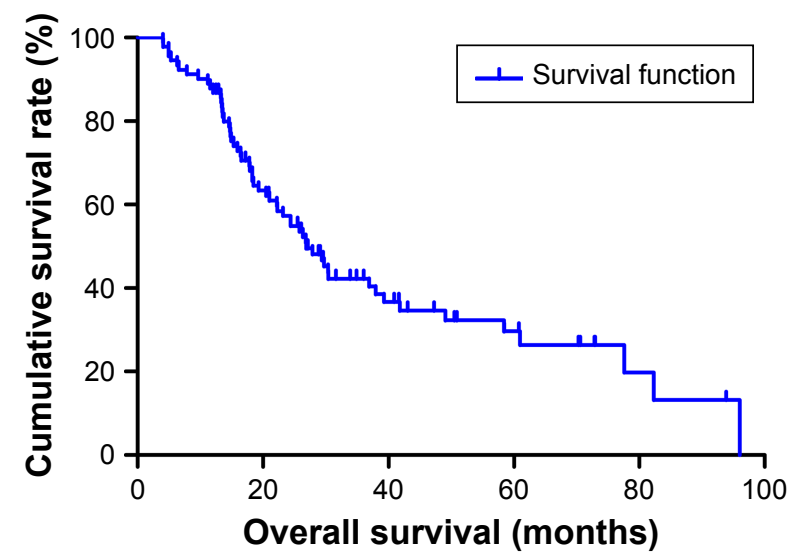

Figure 2 Overall survival of 91 patients with advanced gastric cancer. 
Table 2 Univariate and multivariate Cox regression analyses of disease free survival in 91 patients with advanced gastric cancer

\begin{tabular}{|c|c|c|c|c|}
\hline \multirow[t]{2}{*}{ Parameters } & \multicolumn{2}{|l|}{ Univariate analysis } & \multicolumn{2}{|l|}{ Multivariate analysis } \\
\hline & Hazard ratio $(95 \% \mathrm{Cl})$ & $P$-value & Hazard ratio $(95 \% \mathrm{Cl})$ & $P$-value \\
\hline Age ( $<57$ vs $\geq 57$ years $)$ & $1.024(0.984-1.066)$ & $0.24 I$ & & \\
\hline Gender (male vs female) & $0.799(0.335-1.909)$ & 0.614 & & \\
\hline BMI $\left(<22.32\right.$ vs $\left.\geq 22.32 \mathrm{~kg} / \mathrm{m}^{2}\right)$ & $0.632(0.327-1.219)$ & $0.17 \mid$ & & \\
\hline$A B O$ blood type ( $A$ vs $B$ vs $O$ vs $A B)$ & $1.390(0.899-2.149)$ & 0.138 & & \\
\hline Chemotherapy regimen (SOX vs XELOX) & $1.072(0.508-2.260)$ & 0.856 & & \\
\hline Radical resection ( $R 0$ vs $R I$ vs $R 2$ ) & $2.199(1.365-3.544)$ & 0.001 & $2.059(1.445-2.932)$ & $<0.001$ \\
\hline Type of surgery (distal vs proximal vs total gastrectomy) & $0.755(0.470-1.213)$ & 0.245 & & \\
\hline Differentiation (poorly vs moderately vs well differentiated) & $0.70 \mid(0.375-1.3 \mid 2)$ & 0.267 & & \\
\hline Primary tumor site (upper vs middle vs low $\mathrm{I} / 3$ ) & $0.443(0.218-0.899)$ & 0.024 & & \\
\hline $\begin{array}{l}\text { Pathology (adenocarcinoma vs mucinous vs signet ring } \\
\text { cell carcinoma vs others) }\end{array}$ & $1.366(0.935-1.995)$ & 0.107 & & \\
\hline Clinical T stage (T3 vs T4) & $3.627(0.382-34.475)$ & 0.262 & & \\
\hline Clinical N stage (N0 vs NI vs N2) & $1.122(0.660-1.906)$ & 0.671 & & \\
\hline Clinical TNM stage (II vs III) & $0.183(0.008-4.259)$ & 0.290 & & \\
\hline Pathological T stage (T0 vs TI vs T2 vs T3 vs T4) & $1.076(0.620-1.868)$ & 0.794 & & \\
\hline Pathological N stage (N0 vs NI vs N2 vs N3) & $1.265(0.677-2.363)$ & 0.461 & & \\
\hline Pathological TNM stage (Tis vs I vs II vs III vs IV) & $2.747(1.156-6.526)$ & 0.022 & $2.782(1.829-4.233)$ & $<0.001$ \\
\hline Total lymph nodes ( $<27$ vs $\geq 27$ ) & $0.688(0.348-|.36|)$ & 0.283 & & \\
\hline Positive lymph nodes ( $<3$ vs $\geq 3$ ) & $1.402(0.296-6.640)$ & 0.671 & & \\
\hline HER-2 (0-+ vs ++-+++$)$ & $1.773(0.946-3.323)$ & 0.074 & & \\
\hline Monocyte $\left(<0.44\right.$ vs $\left.\geq 0.44 \times 10^{9} / L\right)$ & $0.665(0.275-1.608)$ & 0.365 & & \\
\hline Lymphocyte ( $<1.68$ vs $\left.\geq 1.68 \times 10^{9} / \mathrm{L}\right)$ & $1.621(0.725-3.627)$ & 0.240 & & \\
\hline MLR $(<0.27$ vs $\geq 0.27)$ & $0.969(0.434-2.165)$ & 0.938 & & \\
\hline Response (CR vs PR vs SD vs PD) & $0.962(0.563-1.644)$ & 0.887 & & \\
\hline
\end{tabular}

Abbreviations: $\mathrm{Cl}$, confidence interval; BMI, body mass index; SOX, S-I plus oxaliplatin; XELOX, oxaliplatin and capecitabine; TNM, tumor-node-metastasis; MLR, monocyte to lymphocyte ratio; CR, complete response; PR, partial response; SD, stable disease; PD, progression of disease; Tis, tumor in situ.

Table 3 Univariate and multivariate Cox regression analyses of overall survival in 9l patients with advanced gastric cancer

\begin{tabular}{|c|c|c|c|c|}
\hline \multirow[t]{2}{*}{ Parameters } & \multicolumn{2}{|l|}{ Univariate analysis } & \multicolumn{2}{|l|}{ Multivariate analysis } \\
\hline & Hazard ratio $(95 \% \mathrm{Cl})$ & $P$-value & Hazard ratio $(95 \% \mathrm{Cl})$ & $P$-value \\
\hline Age ( $<57$ vs $\geq 57$ years $)$ & $1.021(0.980-1.064)$ & 0.312 & & \\
\hline Gender (male vs female) & $1.053(0.447-2.483)$ & 0.906 & & \\
\hline BMI $(<22.32$ vs $\geq 22.32)$ & $0.523(0.263-1.042)$ & 0.065 & & \\
\hline$A B O$ blood type ( $A$ vs $B$ vs $O$ vs $A B)$ & $1.069(0.718-1.592)$ & 0.743 & & \\
\hline Chemotherapy regimen (SOX vs XELOX) & $1.362(0.630-2.946)$ & 0.432 & & \\
\hline Radical resection (R0 vs RI vs R2) & $3.072(1.886-5.005)$ & $<0.001$ & $2.494(1.730-3.595)$ & $<0.001$ \\
\hline Type of surgery (distal vs proximal vs total gastrectomy) & $0.673(0.413-1.096)$ & 0.112 & & \\
\hline Differentiation (poorly vs moderately vs well differentiated) & $0.940(0.503-1.759)$ & 0.847 & & \\
\hline Primary tumor site (upper vs middle vs low I/3) & $0.536(0.259-1.110)$ & 0.093 & & \\
\hline $\begin{array}{l}\text { Pathology (adenocarcinoma vs mucinous vs signet ring cell } \\
\text { carcinoma vs others) }\end{array}$ & $1.292(0.862-1.937)$ & 0.215 & & \\
\hline Clinical T stage (T3 vs T4) & $3.538(0.342-36.638)$ & 0.289 & & \\
\hline Clinical N stage (N0 vs NI vs N2) & $1.065(0.618-1.836)$ & 0.821 & & \\
\hline Clinical TNM stage (II vs III) & $0.139(0.006-3.504)$ & 0.231 & & \\
\hline Pathological $\mathrm{T}$ stage (T0 vs $\mathrm{TI}$ vs $\mathrm{T} 2$ vs $\mathrm{T} 3$ vs $\mathrm{T} 4$ ) & $1.091(0.631-1.884)$ & 0.756 & & \\
\hline Pathological N stage (N0 vs NI vs N2 vs N3) & $1.189(0.640-2.208)$ & 0.581 & & \\
\hline Pathological TNM stage (Tis vs I vs II vs III vs IV) & $2.598(1.057-6.385)$ & 0.037 & $3.04 I(1.949-4.746)$ & $<0.001$ \\
\hline Total lymph nodes ( $<27$ vs $\geq 27$ ) & $0.566(0.287-1.115)$ & 0.100 & & \\
\hline Positive lymph nodes (<3 vs $\geq 3$ ) & $1.799(0.369-8.782)$ & 0.468 & & \\
\hline HER-2 (0-+ vs ++-+++) & $1.565(0.852-2.873)$ & 0.149 & & \\
\hline Monocyte $\left(<0.44\right.$ vs $\left.\geq 0.44 \times 10^{9} / \mathrm{L}\right)$ & $0.191(0.216-1.357)$ & 0.191 & & \\
\hline Lymphocyte $\left(<1.68\right.$ vs $\left.\geq 1.68 \times 10^{9} / \mathrm{L}\right)$ & $1.683(0.743-3.812)$ & 0.212 & & \\
\hline $\operatorname{MLR}(<0.27$ vs $\geq 0.27)$ & $1.054(0.448-2.484)$ & 0.904 & & \\
\hline Response (CR vs PR vs SD vs PD) & $1.038(0.601-1.793)$ & 0.893 & & \\
\hline
\end{tabular}

Abbreviations: $\mathrm{Cl}$, confidence interval; BMI, body mass index; SOX, S-I plus oxaliplatin; XELOX, oxaliplatin and capecitabine; TNM, tumor-node-metastasis; MLR, monocyte to lymphocyte ratio; CR, complete response; PR, partial response; SD, stable disease; PD, progression of disease; Tis, tumor in situ. 


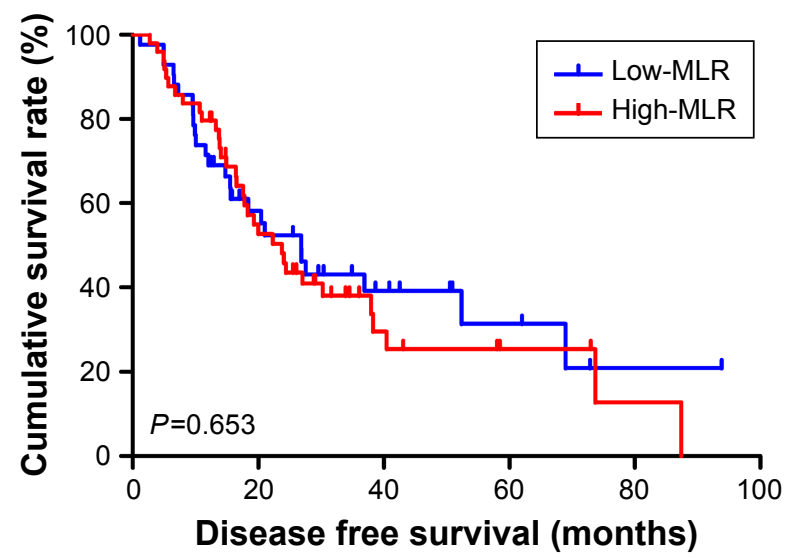

Figure 3 Disease free survival in relation to MLR. Abbreviation: MLR, monocyte to lymphocyte ratio.

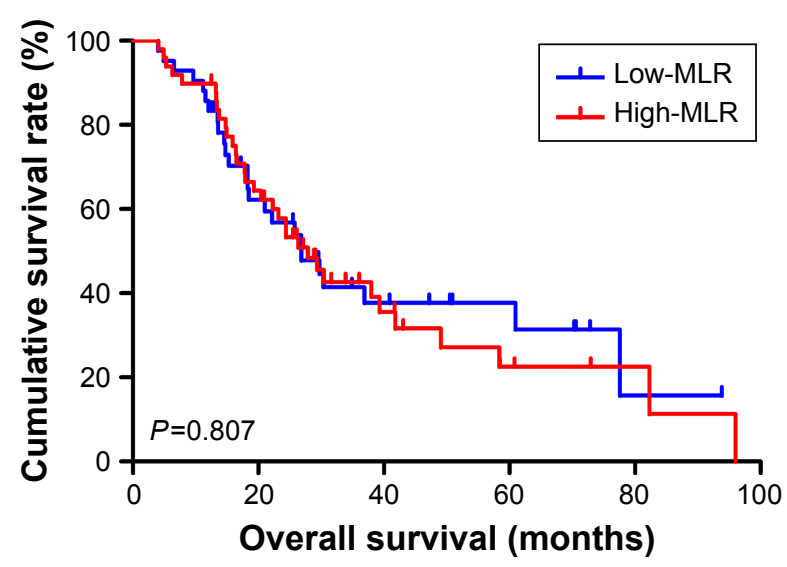

Figure 4 Overall survival in relation to MLR.

Abbreviation: MLR, monocyte to lymphocyte ratio.

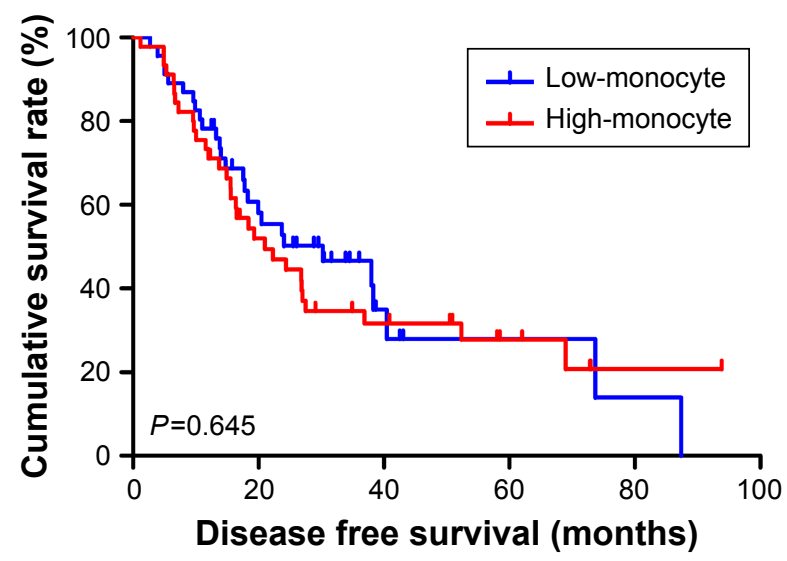

Figure 5 Disease free survival in relation to monocyte count.

The most common toxicities after neoadjuvant chemotherapy were hematologic toxicities. The National Cancer Institute Common Toxicity Criteria grade 1 and 2 anemia, leucopenia, neutropenia, thrombocytopenia were recorded in 33/91 (36.3\%), 18/91 (19.8\%), 21/91 (23.1\%), and 4/91 (4.4\%) of patients respectively (Tables 8 and 9). MLR had

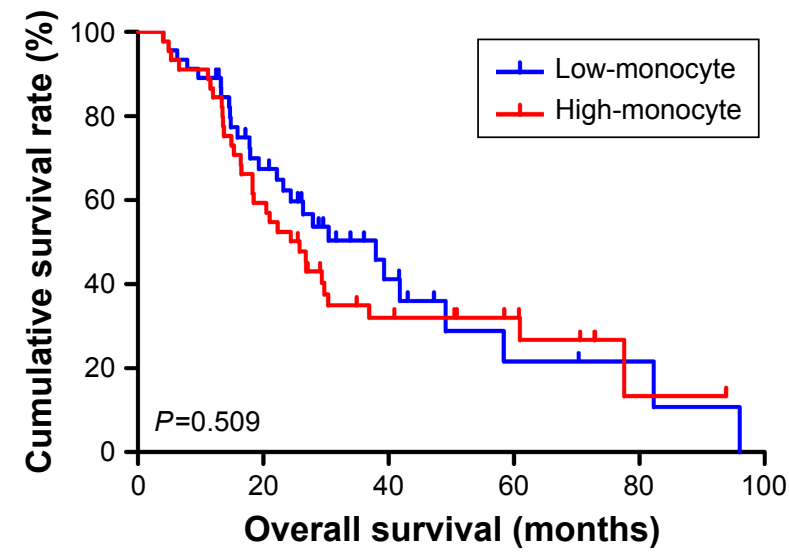

Figure 6 Overall survival in relation to monocyte count.

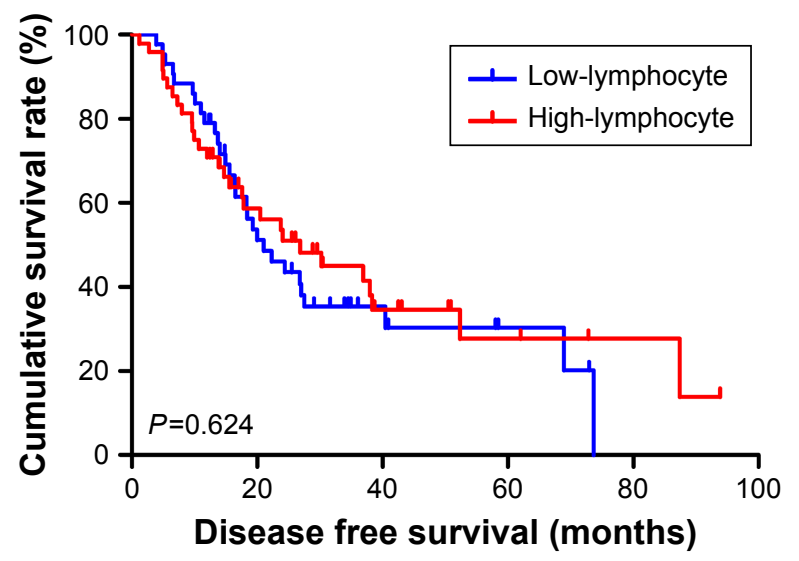

Figure 7 Disease free survival in relation to lymphocyte count.

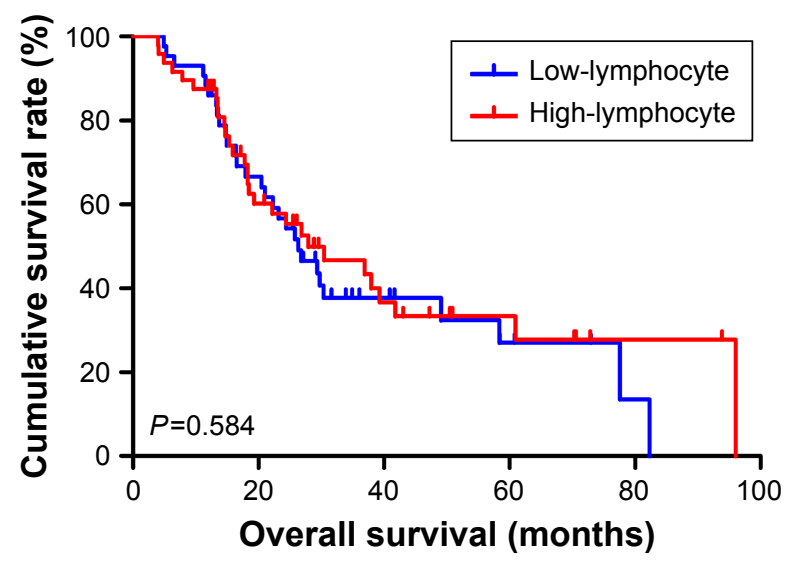

Figure 8 Overall survival in relation to lymphocyte count.

no significance using the cut-off value of 0.27 on toxicities of all patients.

\section{Discussion}

Gastric cancer is one of the most common types of malignant tumors all over the world. The mortality and morbidity rates 
Table 4 Clinical and laboratory characteristics as well as disease free survival and overall survival of the 91 patients with advanced gastric cancer

\begin{tabular}{|c|c|c|c|c|c|c|c|}
\hline Parameters & $\begin{array}{l}\text { Patient } \\
\text { cases (\%) }\end{array}$ & $\begin{array}{l}\text { Disease free survival } \\
\text { (months) }{ }^{a}\end{array}$ & $\chi^{2}$ & $P$-value ${ }^{b}$ & $\begin{array}{l}\text { Overall survival } \\
\text { (months) }\end{array}$ & $\chi^{2}$ & $P$-value \\
\hline Monocyte & & & 0.212 & 0.645 & & 0.437 & 0.509 \\
\hline$<0.44 \times 10^{9} / \mathrm{L}$ & 45 (49.5\%) & $30.23(11.40-49.06)$ & & & $37.97(23.90-52.04)$ & & \\
\hline$\geq 0.44 \times 10^{9} / \mathrm{L}$ & $46(50.5 \%)$ & $21.03(11.42-30.64)$ & & & $25.83(19.08-32.58)$ & & \\
\hline Lymphocyte & & & 0.241 & 0.624 & & 0.300 & 0.584 \\
\hline$<1.68 \times 10^{9} / \mathrm{L}$ & 43 (47.3\%) & $21.03(13.75-28.31)$ & & & $26.37(19.15-33.58)$ & & \\
\hline$\geq 1.68 \times 10^{9} / \mathrm{L}$ & $48(52.7 \%)$ & $26.87(9.89-43.86)$ & & & $27.93(12.21-43.65)$ & & \\
\hline MLR & & & 0.202 & 0.653 & & 0.059 & 0.807 \\
\hline$<0.27$ & $42(46.2 \%)$ & $26.80(17.57-36.04)$ & & & $27.93(20.99-34.87)$ & & \\
\hline$\geq 0.27$ & $49(53.8 \%)$ & $23.73(17.23-30.23)$ & & & $26.87(16.88-36.86)$ & & \\
\hline
\end{tabular}

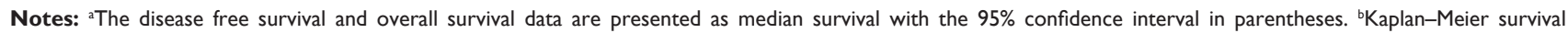
analysis.

Abbreviation: MLR, monocyte to lymphocyte ratio.

Table 5 Clinical and laboratory characteristics as well as disease free survival of the 91 patients with advanced gastric cancer by MLR

\begin{tabular}{|c|c|c|c|c|c|c|}
\hline Parameters & $\mathbf{N}^{\mathbf{a}}$ & Low-MLR $<0.27$ & $\mathbf{N}^{\mathbf{a}}$ & High-MLR $\geq 0.27$ & $\chi^{2}$ & $P$-value \\
\hline Monocyte & & & & & 0.785 & 0.376 \\
\hline$<0.44 \times 10^{9} / \mathrm{L}$ & $30(71.4 \%)$ & $36.30(23.65-48.95)$ & 15 (30.6\%) & $33.05(19.42-46.68)$ & & \\
\hline$\geq 0.44 \times 10^{9} / \mathrm{L}$ & $12(28.6 \%)$ & $31.47(22.85-40.09)$ & 34 (69.4\%) & $33.75(22.47-45.02)$ & & \\
\hline Lymphocyte & & & & & 0.164 & 0.686 \\
\hline$<1.68 \times 10^{9} / \mathrm{L}$ & $13(31.0 \%)$ & $18.40(7.29-29.51)$ & $30(61.2 \%)$ & $22.33(|2.94-3| .72)$ & & \\
\hline$\geq 1.68 \times 10^{9} / \mathrm{L}$ & $29(69.0 \%)$ & $36.93(8.52-65.34)$ & 19 (38.8\%) & $23.73(15.04-32.42)$ & & \\
\hline
\end{tabular}

Note: ${ }^{a}$ The values are based on the MLR, monocyte count, and lymphocyte count by the the optimal cutoff value by receiver operating characteristic. Abbreviation: MLR, monocyte to lymphocyte ratio.

Table 6 Clinical and laboratory characteristics as well as overall survival of the 91 patients with advanced gastric cancer by MLR

\begin{tabular}{|c|c|c|c|c|c|c|}
\hline Parameters & $\mathbf{N}^{\mathbf{a}}$ & Low-MLR < 0.27 & $\mathbf{N}^{\mathbf{a}}$ & High-MLR $\geq 0.27$ & $\chi^{2}$ & $P$-value \\
\hline Monocyte & & & & & 0.703 & 0.402 \\
\hline$<0.44 \times 10^{9} / \mathrm{L}$ & 30 (7I.4\%) & $48.2 \mathrm{I}(3 \mathrm{I} .22-65.2 \mathrm{I})$ & $15(30.6 \%)$ & $34.66(20.97-48.35)$ & & \\
\hline$\geq 0.44 \times 10^{9} / \mathrm{L}$ & $12(28.6 \%)$ & $39.14(27.24-51.03)$ & $34(69.4 \%)$ & $38.16(27.04-49.28)$ & & \\
\hline Lymphocyte & & & & & 0.101 & 0.751 \\
\hline$<1.68 \times 10^{9} / \mathrm{L}$ & 13 (3I.0\%) & $25.83(8.45-43.21)$ & $30(6 \mathrm{I} .2 \%)$ & $29.37(4.4 I-54.34)$ & & \\
\hline$\geq 1.68 \times 10^{9} / \mathrm{L}$ & $29(69.0 \%)$ & $39.93(0-77.04)$ & $19(38.8 \%)$ & $27.93(15.01-40.86)$ & & \\
\hline
\end{tabular}

Note: ${ }^{2}$ The values are based on the MLR, monocyte count, and lymphocyte count by the optimal cutoff value by receiver operating characteristic.

Abbreviation: MLR, monocyte to lymphocyte ratio.

Table 7 The I-year, 3-year, and 5-year DFS and OS rates of the 91 patients with advanced gastric cancer

\begin{tabular}{|c|c|c|c|c|c|c|c|}
\hline \multirow[t]{2}{*}{ Parameters } & \multirow{2}{*}{$\begin{array}{l}\text { Patients } \\
\text { (n) }\end{array}$} & \multicolumn{3}{|l|}{ DFS } & \multicolumn{3}{|l|}{ OS } \\
\hline & & I-year & 3-year & 5-year & I-year & 3-year & 5-year \\
\hline Low-MLR $<0.27$ & 42 & $71.4 \%(30 / 42)$ & $26.2 \%(11 / 42)$ & $9.5 \%(4 / 42)$ & $85.7 \%(36 / 42)$ & $26.2 \%(\mathrm{II} / 42)$ & $14.3 \%(6 / 42)$ \\
\hline High-MLR $\geq 0.27$ & 49 & $79.6 \%$ (39/49) & $20.4 \%(10 / 49)$ & $6.1 \%(3 / 49)$ & $89.8 \%$ (44/49) & $26.5 \%(13 / 49)$ & $8.2 \%(4 / 49)$ \\
\hline Total & 91 & $75.8 \%(69 / 91)$ & $23.1 \%(2|/ 9|)$ & $7.7 \%(7 / 91)$ & $87.9 \%(80 / 91)$ & $26.4 \%(24 / 91)$ & $11.0 \%(10 / 91)$ \\
\hline
\end{tabular}

Abbreviations: DFS, disease free survival; OS, overall survival; MLR, monocyte to lymphocyte ratio. 
Table 8 Main toxicities according to $\mathrm{NCl}-\mathrm{CTC}$ scale of the 91 patients with advanced gastric cancer

\begin{tabular}{|c|c|c|c|c|c|c|c|}
\hline \multirow[t]{2}{*}{ Toxicity } & \multirow{2}{*}{$\begin{array}{l}\text { Patients } \\
\text { (n) }\end{array}$} & \multicolumn{3}{|l|}{ Anemia } & \multicolumn{3}{|l|}{ Leucopenia } \\
\hline & & Grade 0 (\%) & Grade I-2 (\%) & Grade 3-4 (\%) & Grade 0 (\%) & Grade I-2 (\%) & Grade 3-4 (\%) \\
\hline Low-MLR <0.27 & 42 & 27 (64.3\%) & 15 (35.7\%) & $0(0 \%)$ & 30 (7I.4\%) & $12(28.6 \%)$ & $0(0 \%)$ \\
\hline High-MLR $\geq 0.27$ & 49 & 31 (63.3\%) & 18 (36.7\%) & $0(0 \%)$ & 43 (87.6\%) & $6(12.4 \%)$ & $0(0 \%)$ \\
\hline Total & 91 & $58(63.7 \%)$ & 33 (36.3\%) & $0(0 \%)$ & $73(80.2 \%)$ & 18 (19.8\%) & $0(0 \%)$ \\
\hline
\end{tabular}

Abbreviations: NCl-CTC, National Cancer Institute Common Toxicity Criteria; MLR, monocyte to lymphocyte ratio.

of gastric cancer have declined in recent decades, however, it still has a poor prognosis and leads to hundreds of thousands of deaths annually. ${ }^{14}$ Over the past 2 decades, with the rapid advances in surgical techniques and adjuvant therapy, survival and quality of life have been greatly improved for these patients with advanced gastric carcinoma. ${ }^{15}$ Some immunological and histological biomarkers may influence the prognosis of patients with gastric cancer, which largely depends on the primary tumor samples, however, they are often time-consuming and expensive, which limits their use in clinical practice. ${ }^{16}$ Therefore, we should find reliable and affordable prognostic factors in patients with advanced gastric cancer and help the clinicians choose the proper therapies. There is growing interest in a clinical interpretation of the cellular components of a systemic inflammatory response in peripheral venous blood. Nevertheless, the mechanisms by which inflammatory response induces a poor outcome are still ambiguous and poorly understood.

For all we know, a lot of research has indicated that monocyte count is associated with poor survival in patients with many types of cancer, but the potential mechanisms remain unknown. ${ }^{17}$ The monocytes inhibit the immune system and promote tumor proliferation, angiogenesis, and progression..$^{18}$ Furthermore, monocytes can inhibit the immune system by releasing some cytokines and chemokines and regulates tumor microenvironment. The lymphocytes are known to play essential roles in defense of tumor cells and reflect the inability of the body to mediate effective cell-mediated immunity to initiate tumor cell death. ${ }^{19}$ More often than not, increased lymphocyte levels are associated with better prognosis in some solid tumors. ${ }^{20}$ Combined with these results, we found that an increase in the monocyte count and decrease of the lymphocyte count in the peripheral venous blood have been related to tumor growth and progression. Therefore, the MLR may be a good marker to reflect the degree of tumor progression and predict prognosis.

The clinicopathological database of the 91 enrolled patients with advanced gastric cancer was analyzed. Low MLR level may help improve the demographic and clinicopathological characteristics, including gender, primary tumor site, monocyte, and lymphocyte. And improved DFS and OS factors were predicted for R0 resection and pathological Tis/I stage. We found that low MLR, low monocyte, and high lymphocyte were all associated with better prognosis in advanced gastric cancer patients. Meanwhile, our study indicated that low level MLR and low level neutrophil or high level lymphocyte correlated with better median DFS and OS for all patients. The 5-year DFS and OS rates of patients with low level MLR were higher than those of patients with high level MLR. The MLR value lost its independent prognostic significance for DFS or OS, however, it still provided important information for clinical practice.

As far as we know, the MLR values with regard to DFS and OS in patients with advanced gastric cancer undergoing neoadjuvant chemotherapy are rarely reported. The present study indicates that the MLR level may be used to predict the prognosis of advanced gastric cancer patients. It is of importance to take into consideration the high gastric cancer morbidity and unbalanced medical condition in China, and these convenient and cheap biomarkers may be useful for the prevention and treatment of gastric cancer. Hence, a better understanding of hematologic parameters may help identify

Table 9 Main toxicities according to $\mathrm{NCl}-\mathrm{CTC}$ scale of the $9 \mathrm{I}$ patients with advanced gastric cancer

\begin{tabular}{|c|c|c|c|c|c|c|c|}
\hline \multirow[t]{2}{*}{ Toxicity } & \multirow{2}{*}{$\begin{array}{l}\text { Patients } \\
\text { (n) }\end{array}$} & \multicolumn{3}{|l|}{ Neutropenia } & \multicolumn{3}{|c|}{ Thrombocytopenia } \\
\hline & & Grade 0 (\%) & Grade I-2 (\%) & Grade 3-4 (\%) & Grade 0 (\%) & Grade I-2 (\%) & Grade 3-4 (\%) \\
\hline Low-MLR $<0.27$ & 42 & $26(61.9 \%)$ & 14 (33.3\%) & $2(4.8 \%)$ & $4 \mathrm{l}(97.6 \%)$ & I (2.4\%) & $0(0 \%)$ \\
\hline High-MLR $\geq 0.27$ & 49 & $4 \mathrm{I}(83.7 \%)$ & 7 (I4.3\%) & I (2.0\%) & 46 (93.9\%) & $3(6.1 \%)$ & $0(0 \%)$ \\
\hline Total & 91 & 67 (73.6\%) & 21 (23.1\%) & $3(3.3 \%)$ & 87 (95.6\%) & $4(4.4 \%)$ & $0(0 \%)$ \\
\hline
\end{tabular}

Abbreviations: $\mathrm{NCl}-\mathrm{CTC}$, National Cancer Institute Common Toxicity Criteria; MLR, monocyte to lymphocyte ratio. 
new targets for personal treatment. Therefore, this study may provide fundamental information for clinical practice.

To sum up, our study explains the reason for elevated MLR promoting tumor progression, and that low level MLR may represent a more beneficial prognosis. However, the number of patients was small, and larger numbers of patients with advanced gastric cancer treated with neoadjuvant chemotherapy should be enrolled. The differences in the cut-off values of MLR among the studies may be due to the differences in the number of patients and disease stage. In our research, whether the cut-off value of 0.27 for MLR is correct needs further investigation.

\section{Conclusion}

In conclusion, MLR may be a convenient, easily measured prognostic indicator for patients with advanced gastric cancer treated with neoadjuvant chemotherapy. Low level MLR may help clinicians to identify those patients who will benefit from neoadjuvant chemotherapy. However, further investigations are needed to evaluate changes in inflammatory markers in larger groups of patients with advanced gastric cancer.

\section{Disclosure}

The authors report no conflicts of interest in this work.

\section{References}

1. Siegel RL, Miller KD, Jemal A. Cancer statistics, 2015. CA Cancer J Clin. 2015;65(1):5-29.

2. Park SC, Chun HJ. Chemotherapy for advanced gastric cancer: review and update of current practices. Gut Liver. 2013;7(4):385-393.

3. Pera M, Gallego R, Montagut C, et al. Phase II trial of preoperative chemoradiotherapy with oxaliplatin, cisplatin, and 5-FU in locally advanced esophageal and gastric cancer. Ann Oncol. 2012;23(3): 664-670.

4. Bang YJ, Kim YW, Yang HK, et al. Adjuvant capecitabine and oxaliplatin for gastric cancer after D2 gastrectomy (CLASSIC): a phase 3 open-label, randomised controlled trial. Lancet. 2012;379(9813):315-321.
5. Quéro L, Guillerm S, Hennequin C. Neoadjuvant or adjuvant therapy for gastric cancer. World J Gastrointest Oncol. 2015;7(8):102-110.

6. el Aziz LM. Blood neutrophil-lymphocyte ratio predicts survival in locally advanced cancer stomach treated with neoadjuvant chemotherapy FOLFOX 4. Med Oncol. 2014;31(12):311.

7. Grivennikov SI, Greten FR, Karin M. Immunity, inflammation, and cancer. Cell. 2010;140(6):883-899.

8. Balkwill F, Mantovani A. Inflammation and cancer: back to Virchow? Lancet. 2001;357(9255):539-545.

9. Lee S, Oh SY, Kim SH, et al. Prognostic significance of neutrophil lymphocyte ratio and platelet lymphocyte ratio in advanced gastric cancer patients treated with FOLFOX chemotherapy. BMC Cancer. 2013;13:350.

10. Mohri Y, Tanaka K, Ohi M, et al. Identification of prognostic factors and surgical indications for metastatic gastric cancer. BMC Cancer. 2014; 14:409.

11. Feng F, Sun L, Zheng G, et al. Low lymphocyte-to-white blood cell ratio and high monocyte-to-white blood cell ratio predict poor prognosis in gastric cancer. Oncotarget. 2017;8(3):5281-5291.

12. Washington $\mathrm{K}$. 7th edition of the AJCC cancer staging manual: stomach. Ann Surg Oncol. 2010;17(12):3077-3079.

13. Eisenhauer EA, Therasse P, Bogaerts J, et al. New response evaluation criteria in solid tumours: revised RECIST guideline (version 1.1). Eur J Cancer. 2009;45(2):228-247.

14. Fock KM. Review article: the epidemiology and prevention of gastric cancer. Aliment Pharmacol Ther. 2014;40(3):250-260.

15. Brenkman HJ, Haverkamp L, Ruurda JP, van Hillegersberg R. Worldwide practice in gastric cancer surgery. World $J$ Gastroenterol. 2016;22(15):4041-4048.

16. Shirai O, Ohmiya N, Taguchi A, et al. P53, P21 and P73 gene polymorphisms in gastric carcinoma. Hepatogastroenterology. 2010;57(104): 1595-1601.

17. Eo WK, Jeong DW, Chang HJ, et al. Absolute monocyte and lymphocyte count prognostic score for patients with gastric cancer. World $J$ Gastroenterol. 2015;21(9):2668-2676.

18. Gabrilovich DI, Nagaraj S. Myeloid-derived suppressor cells as regulators of the immune system. Nat Rev Immunol. 2009;9(3):162-174.

19. Milne K, Alexander C, Webb JR, et al. Absolute lymphocyte count is associated with survival in ovarian cancer independent of tumorinfiltrating lymphocytes. $J$ Transl Med. 2012;10:33.

20. Quigley DA, Kristensen V. Predicting prognosis and therapeutic response from interactions between lymphocytes and tumor cells. Mol Oncol. 2015;9(10):2054-2062.
OncoTargets and Therapy

\section{Publish your work in this journal}

OncoTargets and Therapy is an international, peer-reviewed, open access journal focusing on the pathological basis of all cancers, potential targets for therapy and treatment protocols employed to improve the management of cancer patients. The journal also focuses on the impact of management programs and new therapeutic agents and protocols on

\section{Dovepress}

patient perspectives such as quality of life, adherence and satisfaction. The manuscript management system is completely online and includes a very quick and fair peer-review system, which is all easy to use. Visit http://www.dovepress.com/testimonials.php to read real quotes from published authors. 